\title{
Impact of Degrees of Freedom of Polyatomic Ideal Gas on Efficiency of Three-Stroke-Cycle Heat Engines
}

\author{
Haiduke Sarafian \\ University College, The Pennsylvania State University, York, PA, USA \\ Email: has2@psu.edu
}

How to cite this paper: Sarafian, H. (2018) Impact of Degrees of Freedom of Polyatomic Ideal Gas on Efficiency of ThreeStroke-Cycle Heat Engines. Journal of Modern Physics, 9, 1807-1813.

https://doi.org/10.4236/jmp.2018.99114

Received: July 16, 2018

Accepted: August 6, 2018

Published: August 9, 2018

Copyright $\odot 2018$ by author and Scientific Research Publishing Inc. This work is licensed under the Creative Commons Attribution International License (CC BY 4.0).

http://creativecommons.org/licenses/by/4.0/

\section{c) (i) Open Access}

\begin{abstract}
It is customary to evaluate the efficiency of four-stroke-cycle heat engines such as Carnot, Otto and Diesel with a working ideal gas [1] [2] [3]. Here we consider various three-stroke-cycle heat engines that are composed of three out of four special processes: adiabatic, isothermal, isobaric and isochoric. We deviate from the customary norm considering the working material to be a polyatomic ideal gas with various degrees of freedom. We develop analytic expressions for the efficiency of each design as a function of degrees of freedom. For suitable practical values of relevant $(V, P, T)$, we evaluate the corresponding efficiencies.
\end{abstract}

\section{Keywords}

Efficiency of Heat Engine, Three-Stroke-Cycle, Adiabatic, Isothermal Processes

\section{Introduction}

Because of the essential conceptual and practical importance of the Carnot heat engine [1] that is conducive to introducing fundamental quantities such as entropy and efficiency, one wrongfully has the misconception that a four-stroke-cycle is the keystone for evaluating the latter. Consequently, a systematic investigation of characteristics of three-stroke-cycle engines is overlooked [1] [2] [3] [4] [5]. As far as the efficiency of engines is concerned, one would think the simplest cycle is the one that is composed of three interlocked rather than four states that are sequentially connected via various thermodynamic processes. Recognizing the void, in this note we consider various three-stroke-cycles composed of three out of four special practical thermodynamic processes namely: adiabatic, iso- 
thermal, isobaric and isochoric. For the working material, we consider polyatomic ideal gases. Given the fact that working materials have different degrees of freedom, we formulate the efficiency of each engine as a function of the corresponding properties. Properties of each gas are specified by: volume, pressure, temperature and degrees of freedom, $(V, P, T, f)$, respectively. For a set of practical parameters, we evaluate their efficiencies. This article is composed of three sections. In addition to Introduction, in Section 2, we outline the physics of the problem providing its solution. Section 3 is the Conclusions with comments on how the investigation may be extended.

\section{Physics of the Problem and Its Solution}

In a V-P diagram a heat engine is shown by a closed loop, see Figure 1. The efficiency is the ratio of the mechanical work done by the cycle i.e. the value of the enclosed area to the intake heat, $\bar{Q}$; direction of the arrow indicates intake. The simplest physically feasible closed loop is formed using three points/states connected via three processes. Throughout this article for the sake of simplicity for a given cycle frequently we interchange the word "leg" and "process". Because we are interested in four specific processes namely: adiabatic, isothermal, isobaric and isochoric, as essential ancillaries we evaluate their corresponding mechanical work and intake heat. For polyatomic ideal gases the states are specified by $(V, P, T)$; i.e. volume, pressure and temperature, respectively; degrees of freedom, $f$, is implicitly included.

\subsection{Case 1}

We consider a three-stroke-cycle that is composed of a sequence of isothermal expansion followed by an isobaric compression completing the cycle via an isochoric compression. The cycle is shown in the $V$-P diagram, Figure 2.

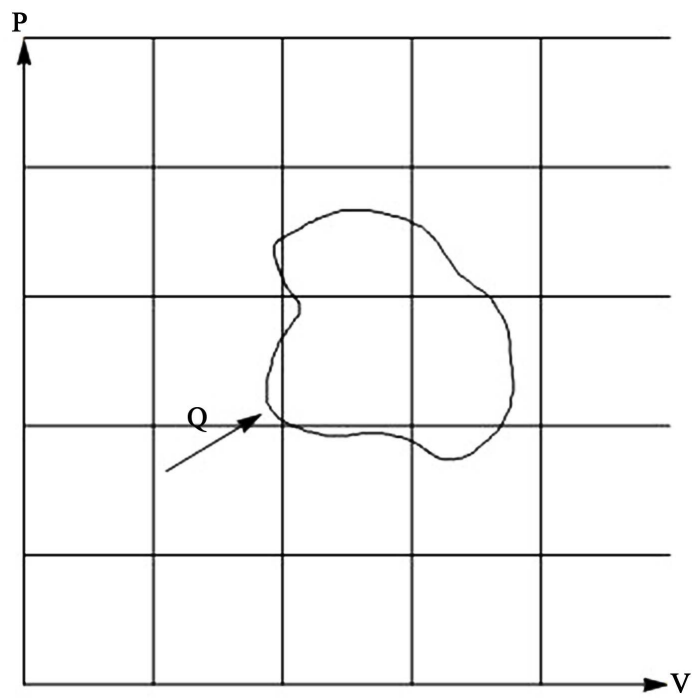

Figure 1. In the shown V-P diagram the closed contour represents a thermodynamic cycle. The enclosed area is the mechanical volumetric work; $\mathrm{Q}$ is the intake heart. 


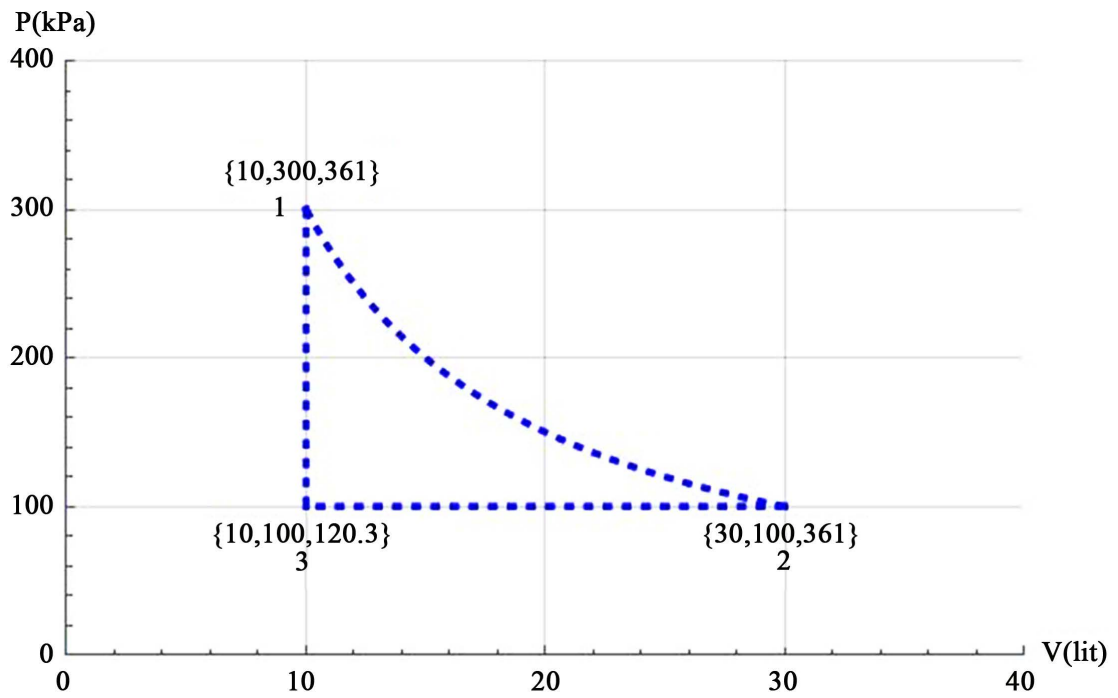

Figure 2. Display of a three-stroke-cycle. Cycle 1231 is composed of isothermal-isobaricisochoric processes. Units of the axes are Volume in liters (lit) and Pressure in Kilo Pascals $(\mathrm{kPa})$.

In Figure 2 the corners of the cycle are labeled $\{1,2,3\}$. Each corner represents the state of the gas according to $\{V, P, T\}$ format. For ideal gases these three quantities are related via Equation of state. The equation of state for one mole of an ideal gas is, $P V=R T, R$ is the ideal gas constant. In Figure 2 specific numeric values are used. For instance, vertex 1 is labeled $\{10,300,361\}$; these stand for 10 lit volume, $300 \mathrm{kPa}$ pressure and $361 \mathrm{~K}$ temperature, respectfully. These values may be adjusted on a need basis. The net mechanical work for the shown cycle is,

$$
W_{1231}=W_{12}+W_{23}
$$

where, the volumetric mechanical work is, $W_{12}=\int_{1}^{2} P \mathrm{~d} V$. Utilizing the ideal gas equation of state yields, $W_{12}=R T_{1} \ln \left(V_{2} / V_{1}\right)$.

Similarly, for the change of state from 2 to 3 we have, $W_{23}=P_{2}\left(V_{3}-V_{2}\right)$. The net volumetric work for the cycle is,

$$
W_{1231}=P_{1} V_{1} \ln \left(\frac{V_{2}}{V_{1}}\right)+\left(P_{3} V_{3}-P_{2} V_{2}\right),
$$

The cycle requires two different intake heats, $\bar{Q}_{12}$ and $\bar{Q}_{31}$. For the isothermal and isochoric legs, we have

$$
Q_{12}=P_{1} V_{1} \ln \left(V_{2} / V_{1}\right) \text {, and } Q_{31}=C_{v} \Delta T \equiv f R / 2\left(T_{1}-T_{3}\right)
$$

where for the molar heat capacity of the ideal gas we applied $C_{v}=f R / 2, f$ is the degrees of the freedom. Hence the net intake heat is,

$$
Q_{1231}=P_{1} V_{1} \ln \left(\frac{V_{2}}{V_{1}}\right)+\frac{1}{2} f\left(P_{1} V_{1}-P_{3} V_{3}\right),
$$

Efficiency of the cycle is $e=W_{1231} / Q_{1231}$. Utilizing (1) and (2) we arrive at, 


$$
e=\frac{\ln \left(\frac{V_{2}}{V_{1}}\right)+\frac{P_{3}}{P_{1}}\left(1-\frac{V_{2}}{V_{1}}\right)}{\ln \left(\frac{V_{2}}{V_{1}}\right)+\frac{1}{2} f\left(1-\frac{P_{3}}{P_{1}}\right)} .
$$

That is the efficiency, it is written as an explicit function of the degrees of freedom and the relevant numeric parameters that can be read off the V-P diagram, Figure 2. As an example, utilizing numeric values of the vertices in Figure 2 for two different values of degrees of freedom, monatomic and polyatomic with degrees of freedom, $f=3$ and 8 , respectively Equation (3) yields, $e_{f=3}=$ $20.5 \%$, and $e_{f=8}=11.5 \%$. That is the working materials with polyatomic gases with higher degrees of freedom result less efficiencies. This is because polyatomic gases with higher degrees of freedom do require greater intake heat per degree temperature change. One also notes that the contour of the cycle and consequentially the value of the mechanical work is independent of the degrees of freedom but the intake heat depends on $f$.

\subsection{Case 2}

We consider a three-stroke-cycle that is composed of a sequence of adiabatic expansion followed by an isobaric compression completing the cycle with an isochoric compression. The cycle is shown in the $V$-P diagram, Figure 3.

There are differences between the cycles shown in Figure 3 vs. the one shown in Figure 2. For the former state 1 and 2 are connected via an isothermal process where for the latter the states are connected adiabatically. As noted the isothermal process is independent of the degrees of freedom, where the adiabatic

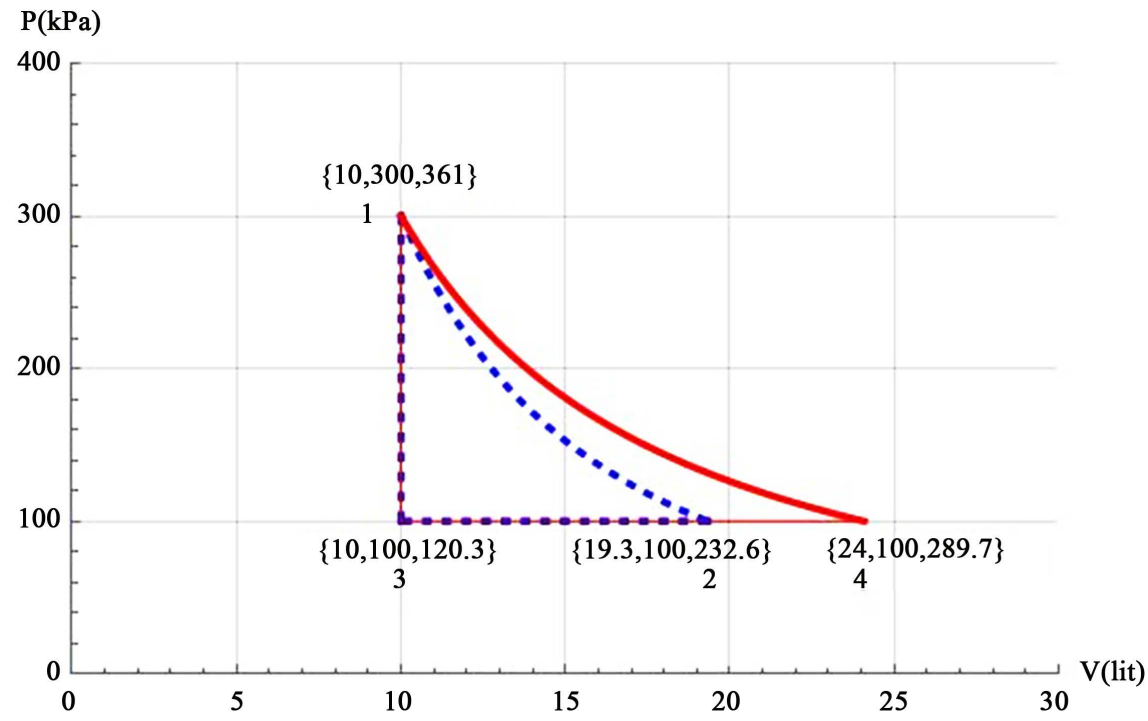

Figure 3. Display of two three-stroke-cycles; cycle 1231 and 1431.Both cycles are composed of adiabatic-isobaric-isochoric processes. Dashed curve corresponds to three degrees of freedom, $f=3$, the solid curve corresponds to eight degrees of freedom, $f=8$. Units of the axes are the same as Figure 2, i.e. Volume in liters (lit) and Pressure in Kilo Pascals $(\mathrm{kPa})$. 
process depends on $f$. Here we evaluate the volumetric value of the mechanical work done via adiabatic process. For ideal gases subject to adiabatic processes it is known [1] the states are related via $P V^{\gamma}=$ const, here $\gamma$ is the adiabatic index and is, $\gamma=C_{P} / C_{V}$ i.e. the ratio of the molar heat capacity at constant pressure to the molar heat capacity at constant volume. Applying Mayer [1] relationship, $C_{P}$ - $C_{V}=R$, and knowing that, $C_{V}=R(1 / 2 f)$, one gets the $\gamma$ in terms of the degrees of freedom namely, $\gamma=1+2 / f$. Applying these relationships in conjunction with equation of state of ideal gases for states related via adiabatic process one obtains additional useful relationships such as: $T V^{1-\gamma}=\frac{C}{R}$ and $T P^{\frac{1}{\gamma}-1}=\frac{c^{\frac{1}{\gamma}}}{R}$. For the volumetric work $w_{12}=\int_{1}^{2} P \mathrm{~d} V$ applying above equations we arrive at, $w_{12}=\frac{C}{1-\gamma}\left(T_{2}-T_{1}\right)$. Utilizing $w_{23}$ from Equation (1) the net volumetric work for the cycle shown in Figure 3 is written as,

$$
w_{1231}=\frac{1}{2} f(P V)_{1}+(P V)_{3}-\left(\frac{1}{2} f+1\right)(P V)_{2},
$$

On the other hand, the intake heat of the cycle is given by Equation (2). Utilizing these we arrive at the efficiency of the cycle,

$$
e=\frac{1}{P_{1}-P_{3}}\left[P_{1}+\frac{2}{f} P_{3}-\left(1+\frac{2}{f}\right) P_{2}\left(\frac{V_{2}}{V_{1}}\right)\right],
$$

As one expects the volumetric mechanical work is a function of the degrees of freedom, yet the intake heat is independent of $f$. Consequently, depending on value of $f$ the contour of the cycle changes accordingly. Two such contours are depicted in Figure 3. The steep dashed curve between state (1) and (2) corresponds to $f=3$, the soft solid curve connecting state (1) to (4) corresponds to $f=$ 8. For two values of degrees of freedom such as, $f=3$ and 8 Equation (5) yields two values of efficiencies, $e_{f=3}=22.5 \%$ and $e_{f=8}=12.5 \%$.

By comparing the efficiencies of the cycles depicted in Figure 2 and Figure 3 we realize their comparative values. It is interesting noting that the impact of the value of $f$ in these two cycles are inverted. For the former the contour of the cycle i.e the volumetric work is $f$ independent, where for the latter it dependents on $f$. On the other hand, the intake heat has baring on the former and has no impact on the latter.

For comprehensive understanding one needs to compare Figure 2 and Figure 3.

\subsection{Case 3}

We consider a three-stroke-cycle that is composed of a sequence of adiabatic expansion followed by an isothermal compression closing the cycle via an isochoric compression. One such cycle is shown in the $V$ - $P$ diagram, Figure 4. 

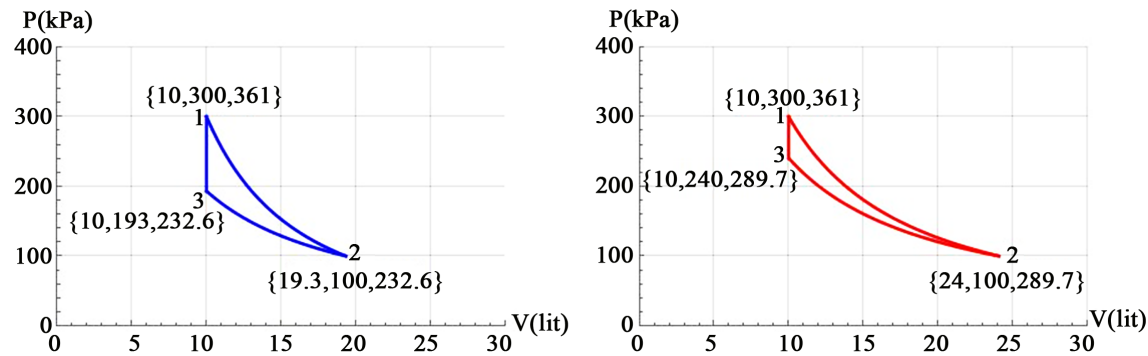

Figure 4. Left cycle clockwise 1231 is composed of adiabatic-isothermal-isometric processes with three degrees of freedom, $f=3$. The right cycle is like the left diagram corresponding to eight degrees of freedom, $f=8$. Units of the axes are the same as Figure 2, i.e. Volume in liters (lit) and Pressure in Kilo Pascals (kPa).

Evaluation of the efficiency of the cycle shown in Figure 4 as a function of degrees of freedom is straight forward. The volumetric mechanical work and intake heat of each process leg of the cycle is given in the previous cases. Utilizing these expressions, the efficiency of the given cycle yields,

$$
e=\frac{(P V)_{1}-(P V)_{2}+\frac{2}{f}(P V)_{2} \ln \left(\frac{V_{3}}{V_{2}}\right)}{V_{1}\left(P_{1}-P_{3}\right)} .
$$

Applying Equation (6) for the numeric values of the vertices shown in Figure 4 , we arrive at the efficiencies, namely, $e_{f=3}=21.0 \%$ and $e_{f \in 8}=12.4 \%$.

\section{Conclusions}

Our investigation fills in the missing systematic information on the efficiency of three-stroke-cycle heat engines. To form a comprehensive understanding, we narrowed our investigation to practical cycles that are composed of three out of four thermodynamic processes: adiabatic, isothermal, isobaric and isobaric. We derived analytic expressions for the efficiency of each designed three-stroke-heat engine. As expected the efficiencies are explicit functions of degrees of freedom of the polyatomic gases. This underlines the fact that working material of the designed engine plays a substantial role. This contrasts with the famous Carnot heat engine where its efficiency is independent of the working material [6]. Applying derived formulations namely, Equations (3) and (5) and (6) to the set of relevant practical numeric $\{V, P, T\}$ values shown in Figures 1-4 we calculate the efficiencies for two distinct values of degrees of freedom. Analyzing these values, we deduce the fact that for our designed three-stroke-heat engines the efficiencies of high and low degrees of freedom are related via, $\mathrm{e}_{\mathrm{f}=8} \sim 0.5 \mathrm{e}_{\mathrm{f}=3}$. While investigating we consider a few more three-stroke-cycles. However, we decided to report the ones that we consider most interesting.

Our investigation sets the stage for extending systematic analysis for four and five-stroke-cycle heat engines conducive to posing questions such as: 1) What is the relationship between the efficiency and the number of the strokes of a cycle? and 2) Is it possible to design a multiple-stroke-cycle heat engine that its efficiency is independent of the working material? 
A note to the readers: All the figures and the implicit calculations in this article are carried out utilizing Mathematica resources [7] [8].

\section{Conflicts of Interest}

The author declares no conflicts of interest regarding the publication of this paper.

\section{References}

[1] Longair, M.S. (1994) Theoretical Concepts in Physics. Cambridge University Press.

[2] Wolfgang, D. and Westfall, G. (2011) University Physics with Modern Physics. McGraw-Hill.

[3] Halliday, D., Resnick, R. and Walkers, J. (2011) Fundamentals of Physics. 9th Edition, John Wiley \& Sons.

[4] Marion, J. and Hornyak, W. (1984) Principle of Physics. Saunders College Publishing.

[5] Tipler, P. and Mosca, G. (2008) Physics for Scientists and Engineers. 6th Edition, W.H. Freeman and Company.

[6] Sarafian, H. (IMS2003) Proceedings of the 5th International Mathematica Symposium, Imperial College London Press, London.

[7] Wolfram, S. (1996) Mathematica Book. 3rd Edition, Cambridge University Press, Cambridge.

[8] Sarafian, H. (2015) Mathematica Graphics Example Book for Beginners. Scientific Research Publishing. 